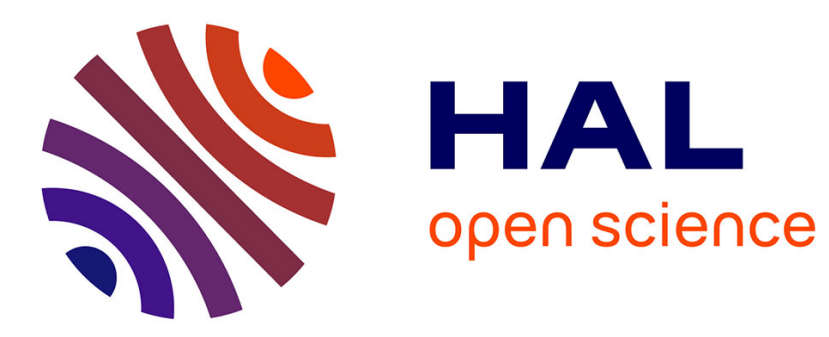

\title{
Graph signal active contours
}

Olivier Lézoray

\section{To cite this version:}

Olivier Lézoray. Graph signal active contours. International Conference on Pattern Recognition (ICPR - IEEE), 2021, Milan, Italy. hal-03107668

\section{HAL Id: hal-03107668 https://hal.science/hal-03107668}

Submitted on 12 Jan 2021

HAL is a multi-disciplinary open access archive for the deposit and dissemination of scientific research documents, whether they are published or not. The documents may come from teaching and research institutions in France or abroad, or from public or private research centers.
L'archive ouverte pluridisciplinaire HAL, est destinée au dépôt et à la diffusion de documents scientifiques de niveau recherche, publiés ou non, émanant des établissements d'enseignement et de recherche français ou étrangers, des laboratoires publics ou privés. 


\title{
Graph signal active contours
}

\author{
Olivier Lézoray \\ Normandie Univ, UNICAEN, ENSICAEN, CNRS, GREYC \\ 14000 Caen, France \\ olivier.lezoray@unicaen.fr
}

\begin{abstract}
With the advent of data living on vertices of graphs, there is much interest in processing the so-called graph signals for partitioning tasks. As active contours have had much impact in the image processing community, their formulation on graphs is of importance to the field of graph signal processing. This paper proposes an adaptation on graphs of a model that combines the Geodesic Active Contour and the Active Contour Without Edges models. In addition, specific terms depending on graphs are introduced in the formulation. This adaptation is solved using a level set formulation with a gradient descent that can be expressed as a morphological front evolution process. Experimental results on different kinds of graphs signals show the benefit of the approach.
\end{abstract}

\section{INTRODUCTION}

Recent technological advances in terms of acquisition processes and digital simulations are currently generating a very large amount of digital data, which may be of different types or natures. This data can come from different application areas, such as digital imaging, complex networks (social, biological or computer), graphical computing or bioinformatics. This data can be, for example, images, videos, meshes, point clouds, networks or databases. On the contrary to classical images and videos, these data do not necessarily lie on a Cartesian grid and can be irregularly distributed. To represent a large number of data domains, the most natural and flexible representation consists in using weighted graphs by modeling neighborhood similarity relationships. Therefore, there is much interest in the transposition of signal and image processing tools for the processing of functions on graphs [1], [2]. An example of such effort include, e.g., spectral graph wavelets [3], [4] or PDEs on graphs [5]-[7]. In this paper, we consider active contour models. They can be casted in two approaches: geometric approaches with level sets and variational approaches with Total Variation. We consider the first approach in this paper and propose an adaptation on graphs that combines and extends the Geodesic Active Contour and the Active Contour Without Edges models.

\section{OPERATORS ON GRAPH SIGNALS}

In this section, we introduce the formulation of a discrete calculus framework for graphs signals [7]. These basic ingredients will be used to adapt active contours for graph signals.

\section{A. Notations}

A graph $\mathcal{G}=(\mathcal{V}, \mathcal{E})$ consists in a finite set $\mathcal{V}=\left\{v_{1}, \ldots, v_{m}\right\}$ of vertices and a finite set $\mathcal{E} \subset \mathcal{V} \times \mathcal{V}$ of edges. We assume $\mathcal{G}$ to be undirected, with no self-loops and no multiple edges.
Let $\left(v_{i}, v_{j}\right)$ be the edge of $\mathcal{E}$ that connects two vertices $v_{i}$ and $v_{j}$ of $\mathcal{V}$. Its weight, denoted by $w_{i j}=w\left(v_{i}, v_{j}\right)$, represents the similarity between its vertices and is computed using a positive symmetric function $w$. The notation $v_{i} \sim v_{j}$ is used to denote two adjacent vertices. The degree of a vertex $v_{i}$ is defined as $\operatorname{deg}\left(v_{i}\right)=\sum_{v_{j} \sim v_{i}} w\left(v_{i}, v_{j}\right)$. Let $\mathcal{H}(\mathcal{V})$ be the Hilbert space of real-valued functions defined on the vertices of a graph. A function $f \in \mathcal{H}(\mathcal{V})$ assigns real-valued vectors $f\left(v_{i}\right)$ to each vertex $v_{i} \in \mathcal{V}$. Such a function is called a graph signal and is represented by the mapping $f: \mathcal{G} \rightarrow \mathbb{R}^{d}$. By analogy with functional analysis on continuous spaces, the integral of a function $f \in \mathcal{H}(\mathcal{V})$, over the set of vertices $\mathcal{V}$, is defined as $\int_{\mathcal{V}} f=\sum_{\mathcal{V}} f$. Similarly, let $\mathcal{H}(\mathcal{E})$ be the space of real-valued functions defined on the edges of $\mathcal{G}$. Both Hilbert spaces are endowed with the usual inner products.

\section{B. Difference operators}

Let $f: \mathcal{V} \rightarrow \mathbb{R}$ be a function of $\mathcal{H}(\mathcal{V})$. The weighted difference operator [6] of $f$, noted $d_{w}: \mathcal{H}(\mathcal{V}) \rightarrow \mathcal{H}(\mathcal{E})$, is defined on an edge $\left(v_{i}, v_{j}\right) \in \mathcal{E}$ by:

$$
\left(d_{w} f\right)\left(v_{i}, v_{j}\right)=w\left(v_{i}, v_{j}\right)^{1 / 2}\left(f\left(v_{j}\right)-f\left(v_{i}\right)\right)
$$

Based on the difference operator, the weighted morphological external and internal difference operators are respectively [8]:

$$
\begin{array}{r}
\left(d_{w}^{+} f\right)\left(v_{i}, v_{j}\right)=\max \left(0,\left(d_{w} f\right)\left(v_{i}, v_{j}\right)\right) \text { and } \\
\left(d_{w}^{-} f\right)\left(v_{i}, v_{j}\right)=-\min \left(0,\left(d_{w} f\right)\left(v_{i}, v_{j}\right)\right)
\end{array}
$$

The adjoint of the difference operator, noted $d_{w}^{*}: \mathcal{H}(\mathcal{E}) \rightarrow$ $\mathcal{H}(\mathcal{V})$, is a linear operator defined, for all $f \in \mathcal{H}(\mathcal{V})$ and all $H \in \mathcal{H}(\mathcal{E})$, by $\left\langle d_{w} f, H\right\rangle_{\mathcal{H}(\mathcal{E})}=\left\langle f, d_{w}^{*} H\right\rangle_{\mathcal{H}(\mathcal{V})}$. This adjoint operator $d_{w}^{*}=-d i v_{w}$, of a function $H \in \mathcal{H}(\mathcal{E})$, can by expressed at a vertex $v_{i} \in \mathcal{V}$ by the following expression:

$$
\left(d_{w}^{*} H\right)\left(v_{i}\right)=\sum_{v_{j} \sim v_{i}} w\left(v_{i}, v_{j}\right)^{1 / 2}\left(H\left(v_{j}, v_{i}\right)-H\left(v_{i}, v_{j}\right)\right) .
$$

\section{Gradients and norms}

The weighted gradient operator of a function $f \in \mathcal{H}(\mathcal{V})$, at a vertex $v_{i} \in \mathcal{V}$, is the vector operator defined by

$$
\left(\nabla_{w} f\right)\left(v_{i}\right)=\left[\left(d_{w} f\right)\left(v_{i}, v_{j}\right): v_{j} \sim v_{i}\right]^{T} .
$$

The $\mathcal{L}_{p}$ norm of this vector is defined by [6]:

$$
\left\|\left(\nabla_{w} f\right)\left(v_{i}\right)\right\|_{p}^{p}=\sum_{v_{j} \sim v_{i}} w\left(v_{i}, v_{j}\right)^{p / 2}\left|f\left(v_{j}\right)-f\left(v_{i}\right)\right|^{p}
$$

Similarly, we have

$$
\left(\nabla_{w}^{ \pm} f\right)\left(v_{i}\right)=\left[\left(d_{w}^{ \pm} f\right)\left(v_{i}, v_{j}\right): v_{j} \sim v_{i}\right]^{T}
$$


and

$$
\left\|\left(\nabla_{w}^{ \pm} f\right)\left(v_{i}\right)\right\|_{p}^{p}=\sum_{v_{j} \sim v_{i}} w\left(v_{i}, v_{j}\right)^{p / 2}\left|M^{ \pm}\left(0, f\left(v_{j}\right)-f\left(v_{i}\right)\right)\right|^{p}
$$

with $M^{+}=\max$ and $M^{-}=\min$. These norms exhibit the following property [9]

$$
\left\|\left(\nabla_{w} f\right)\left(v_{i}\right)\right\|_{p}^{p}=\left\|\left(\nabla_{w}^{+} f\right)\left(v_{i}\right)\right\|_{p}^{p}+\left\|\left(\nabla_{w}^{-} f\right)\left(v_{i}\right)\right\|_{p}^{p} .
$$

\section{Graph signal curvature operator}

The weighted p-Laplace isotropic operator of a function $f \in \mathcal{H}(\mathcal{V})$, noted $\Delta_{w, p}^{i}: \mathcal{H}(\mathcal{V}) \rightarrow \mathcal{H}(\mathcal{V})$, is defined by:

$$
\left(\Delta_{w, p} f\right)\left(v_{i}\right)=\frac{1}{2} d_{w}^{*}\left(\left\|\left(\nabla_{w} f\right)\left(v_{i}\right)\right\|_{2}^{p-2}\left(d_{w} f\right)\left(v_{i}, v_{j}\right)\right) .
$$

The isotropic $p$-Laplace operator of $f \in \mathcal{H}(\mathcal{V})$, at a vertex $v_{i} \in \mathcal{V}$, can be computed by [6]:

$$
\left(\Delta_{w, p} f\right)\left(v_{i}\right)=\frac{1}{2} \sum_{v_{j} \sim v_{i}}\left(\gamma_{w, p} f\right)\left(v_{i}, v_{j}\right)\left(f\left(v_{i}\right)-f\left(v_{j}\right)\right),
$$

with $\left(\gamma_{w, p} f\right)\left(v_{i}, v_{j}\right)=w_{i j}\left(\left\|\left(\nabla_{w} f\right)\left(v_{j}\right)\right\|_{2}^{p-2}+\left\|\left(\nabla_{w} f\right)\left(v_{i}\right)\right\|_{2}^{p-2}\right)$. When $p=1$ this corresponds to the weighted curvature of the graph signal $f:\left(\kappa_{w} f\right)\left(v_{i}\right)=\left(\Delta_{w, 1} f\right)\left(v_{i}\right)$.

\section{ACTIVE CONTOURS}

Now we briefly review the classical Geodesic Active Contour (GAC) [10] and Active Contours Without Edges (ACWE) [11] approaches.

\section{A. Geodesic active contours}

In the GAC framework, an energy functional that depends on the content of an image $I:[0, h] \times[0, w] \rightarrow \mathbb{R}^{d}$ is assigned to a parametrized curve $\mathcal{C}(p):[0,1] \rightarrow \mathbb{R}^{2}$ :

$$
E_{G A C}(C)=\int_{0}^{1} g(I(C(p)))\left|C^{\prime}(p)\right| d p
$$

where $g: \mathbb{R}^{d} \rightarrow \mathbb{R}^{+}$is a strictly decreasing function such that $g(x) \rightarrow 0$ as $x \rightarrow \infty$, e.g., $g(I(x, y))=\frac{1}{\sqrt{1+\left|\nabla G_{\sigma} * I(x, y)\right|}}$ with $G_{\sigma} * I(x, y)$ a smoothed version of $I$ by the Gaussian $G_{\sigma}$. This function allows to select the regions of the image we are interested in. It should have low values for edges and by this way, the curve is attracted by image edges where $g(I)$ is small. The minimum energy $\mathcal{C}^{*}=\arg \min _{C} E_{G A C}(C)$ corresponds to the geodesics of a Riemannian space whose metric is defined by $g(I)$ [12]. To find this geodesic curve, the minimization is done with a steepest descent, that will give a local minimum of Eq. (12), that is not convex. The Euler-Lagrange functional gives the direction of the descent and a local minima is given by

$$
\frac{\delta C}{\delta t}=(g(I) \cdot \kappa+\nabla g(I) \cdot \mathcal{N}) \mathcal{N}
$$

where $\mathcal{N}$ is the curve normal vector and $\kappa$ is the curvature value. This model has been modified in [13] to integrate a balloon force to overcome cases where $g(I)$ is too low:

$$
\frac{\delta C}{\delta t}=(g(I) \cdot \kappa+v g(I)+\nabla g(I) \cdot \mathcal{N}) \mathcal{N}
$$

This equation can be rewritten in the level-set framework as [12]:

$$
\frac{\delta f}{\delta t}=g(I)|\nabla f| \operatorname{div}\left(\frac{\nabla f}{|\nabla f|}\right)+v g(I)|\nabla f|+\nabla g(I) \cdot \nabla f
$$

with $f$ a signed function that embeds the curve, $\kappa=\operatorname{div}\left(\frac{\nabla f}{|\nabla f|}\right)$ the curvature and $v$ a parameter. The first term is called the smoothing force term and is a weighted version (by $g(I)$ ) of the mean curvature motion PDE. The second term is called the balloon force and corresponds to a normal direction motion. The last term is called the attraction force term and corresponds to an external velocity field motion.

\section{B. Active contours without edges}

In the geodesic active contours, the region interior is not considered and no region homogeneity criterion is required: only the image structures under the curve are important to find the solution. To cope with this, Chan and Vese [11] have proposed an energy functional for image segmentation that takes into account the content of the interior and the exterior regions of the curve in contrast to the GAC that takes into account only the places where the curve passes. They called it Active Contours Without Edges (ACWE). In this framework, two regions are separated by a curve $C$. The solution is allowed to have only two values: $c_{1}$ inside $C$ and $c_{2}$ outside, corresponding to a piecewise constant model of the two regions. The AWCE functional of a curve $C$ is

$$
\begin{aligned}
& E_{A C W E}\left(C, c_{1}, c_{2}\right)=\mu \cdot \operatorname{Length}(C)+v \cdot \operatorname{Area}(\operatorname{inside}(C))+ \\
& \quad \lambda_{1} \int_{\operatorname{inside}(C)}\left|I(x)-c_{1}\right|^{2} d x+\lambda_{2} \int_{\operatorname{outside}(C)}\left|I(x)-c_{2}\right|^{2} d x
\end{aligned}
$$

where $\mu, v, \lambda_{1}$ and $\lambda_{2}$ are positive parameters to control the strength of each term and $c_{1}, c_{2}$ are the two region averages. The first term controls the regularity by penalizing the length. The second term penalizes the enclosed area of $C$ to control its size. The last terms penalize the deviation from the piecewise constant approximation of the image. The curve is represented as a level-set of a function $f$ and the minimization is solved by alternating the updates of both $c_{1}$ and $c_{2}$ (the region averages) and then of $f$. First, for a fixed $f, c_{1}$ and $c_{2}$ are updated by computing the region averages. Second, for fixed $c_{1}$ and $c_{2}$, the Euler-Lagrange equation of Eq. (16) gives the direction of the descent and a local minima is expressed as

$$
\frac{\delta f}{\delta t}=\delta_{\varepsilon}(f)\left[\mu \operatorname{div}\left(\frac{\nabla f}{|\nabla f|}\right)-v-\lambda_{1}\left(I-c_{1}\right)^{2}+\lambda_{2}\left(I-c_{2}\right)^{2}\right]
$$

where $\delta_{\varepsilon}$ is defined by $\delta_{\varepsilon}(x)=\frac{\varepsilon}{\pi\left(\varepsilon^{2}+x^{2}\right)}$ that corresponds to the derivative of a regularized Dirac function.

\section{ACTIVE CONTOURS FOR GRAPHS SIGNALS}

In this section we propose an adaptation of active contours for graph signals. Instead of considering separately the GAC and ACWE approaches, we consider an unified active contour formulation that combines both approaches. This is different 
from previous works on active contours on graphs that considered each model separately [14]-[16].

\section{A. Considered active contours}

In [17], it was proposed to consider an energy functional that combines the geodesic active contour and the ChanVese models. In the spirit of this approach, we consider the following energy functional for vector-valued signals of dimension $d$, that has been introduced in [18]:

$$
\begin{gathered}
E\left(\mathcal{C}, c_{1}, c_{2}\right)= \\
\mu \int_{0}^{1} g(C(p))\left|C^{\prime}(p)\right| d p+v \cdot \int_{\operatorname{inside}(C)} g(C(p)) d A+ \\
\frac{\lambda_{1}}{d} \int_{\operatorname{inside}(C)}\left\|I(x)-c_{1}\right\|_{2}^{2} d x+\frac{\lambda_{2}}{d} \int_{\operatorname{outside}(C)}\left\|I(x)-c_{2}\right\|_{2}^{2} d x
\end{gathered}
$$

This can be solved using a gradient descent from the associated Euler-Lagrange equations. We then get the following evolution equation in the level-set framework (by using similar derivation arguments than in [18], [19]):

$$
\begin{aligned}
\frac{\delta f}{\delta t}=\delta_{\varepsilon}(f) & {\left[\mu d i v\left(g(I) \frac{\nabla f}{|\nabla f|}\right)+v \cdot g(I)\right.} \\
- & \left.\frac{\lambda_{1}}{d}\left\|I-c_{1}\right\|_{2}^{2}+\frac{\lambda_{2}}{d}\left\|I-c_{2}\right\|_{2}^{2}\right]
\end{aligned}
$$

that we rewritten and simplify into

$$
\begin{array}{r}
\frac{\delta f}{\delta t}=\delta_{\varepsilon}(f)\left[\mu \cdot g(I) \operatorname{div}\left(\frac{\nabla f}{|\nabla f|}\right)+\mu \nabla g(I) \cdot \nabla f+v \cdot g(I)\right. \\
\left.-\frac{\lambda_{1}}{d}\left\|I-c_{1}\right\|_{2}^{2}+\frac{\lambda_{2}}{d}\left\|I-c_{2}\right\|_{2}^{2}\right]
\end{array}
$$

This provides a formulation similar to the one proposed in [20]. One can see that this model combines the three terms from the GAC with those of the ACWE, leading to a unified formulation. In the sequel, we show how this can be adapted to process signals on weighted graphs.

\section{B. PDEs on graphs}

In [14], we have proposed an adaptation of PDEs on graphs. By analogy with the level-set formulation for the continuous case, an evolving front $\Gamma$ evolving on a graph $\mathcal{G}$ is defined as a subset $\Omega_{0} \subset \mathcal{V}$ and is implicitly represented at time $t$ by a level set function $f_{t}: \mathcal{V} \rightarrow\{-1,+1\}$ defined by $f_{t}=\chi_{\Omega_{t}}-\chi_{\overline{\Omega_{t}}}$ where $\chi: \mathcal{V} \rightarrow\{0,1\}$ is the indicator function and $\overline{\Omega_{t}}$ is the complement of $\Omega_{t}$ (one has $\Omega_{t} \cup \overline{\Omega_{t}}=\mathcal{V}$ ). The evolution of the front $\Gamma$ is influenced by a speed function $\mathcal{F}: \mathcal{V} \rightarrow \mathbb{R}$ in such a way that the propagation direction is controlled by the sign of $\mathcal{F}$. The front extends (i.e., vertices are added to $\Omega_{t}$ ) when this sign is positive and retracts when it is negative (i.e., vertices are removed from $\Omega_{t}$ ). The propagation of the front on the graph can then be described by the following equation

$$
\frac{\delta f\left(v_{i}, t\right)}{\delta t}=\mathcal{F}\left(v_{i}\right)\left\|\left(\nabla_{w} f\right)\left(v_{i}, t\right)\right\|_{p}^{p}
$$

with $f\left(v_{i}, t=0\right)=f_{0}$ (the level-set representation of $\Omega_{0}$ ). Using (9) This evolution equation can then be expressed as a combination of two morphological erosion and dilation processes as [14]:

$$
\begin{aligned}
\frac{\delta f\left(v_{i}, t\right)}{\delta t}= & \max \left(\mathcal{F}\left(v_{i}, t\right), 0\right)\left\|\left(\nabla_{w}^{+} f\right)\left(v_{i}\right)\right\|_{p}^{p}+ \\
& \min \left(\mathcal{F}\left(v_{i}, t\right), 0\right)\left\|\left(\nabla_{w}^{-} f\right)\left(v_{i}\right)\right\|_{p}^{p}
\end{aligned}
$$

that explicitly shows that a dilation adds vertices to the front when the speed is positive and that an erosion removes vertices from the front when the speed is negative.

\section{Adaptation on weighted graphs}

Now we propose an adaptation of Eq. (20) on graphs. Before to do so, we introduce local patches on graphs and use them to define the potential function $g$ on graphs as well as consider patch-based region averages.

1) Local patches on graphs: A local patch $B_{k}\left(v_{i}\right)$ on a graph at a vertex $v_{i}$ is a subgraph of the processed graph. Given a vertex $v_{i}$ and a radius size $k$, the structuring element is composed of all the vertices that can be reached from $v_{i}$ in $k$ walks:

$$
B_{k}\left(v_{i}\right)=\left\{\begin{aligned}
&\left\{v_{i}\right\} \text { if } k=0 \\
&\left\{v_{j} \sim v_{i}\right\} \cup\left\{v_{i}\right\} \text { if } k=1 \\
& B_{k-1}\left(v_{i}\right) \cup\left(\cup_{\forall v_{l} \in B_{k-1}\left(v_{i}\right)} B_{1}\left(v_{l}\right)\right) \text { if } k \geq 2
\end{aligned}\right.
$$

The number of vertices in a given $k$-hop neighborhood $B_{k}\left(v_{i}\right)$ depends on the vertex $v_{i}$ when the graph is irregular. On gridgraphs such as images, $B_{k}\left(v_{i}\right)$ corresponds to a patch centered on $v_{i}$ and is of size $(2 k+1)^{2}$. We will denote by $\mathcal{P}_{k}\left(f, v_{i}\right)$ the set of graph signal values assigned to all the vertices of $B_{k}\left(v_{i}\right)$.

2) Potential function: For graph signals, a vertex can be considered as an important structure if it is different from its neighbors. This amounts to say that the vertex has high spectral distances with its neighbors. In contrast, a vertex in an almost flat area will have low spectral distances with its neighbors. This can be used to construct a potential function that differentiates the most salient structures in the graph signal. We propose to consider a normalized sum of the spectral distances between the vertex $v_{i}$ and its neighbors within $B_{k}\left(v_{i}\right)$ to construct a potential function. It is defined as:

$$
\rho\left(v_{i}\right)=\frac{\sum_{v_{j} \in B_{k}\left(v_{i}\right)} d\left(\mathcal{P}_{\alpha}\left(f, v_{j}\right), \mathcal{P}_{\alpha}\left(f, v_{i}\right)\right)}{\left|B_{k}\left(v_{i}\right)\right|}
$$

with $\alpha<k$ and $d$ a distance to compare the values of two patches.

$$
g\left(v_{i}\right)=1-\frac{\rho\left(v_{i}\right)-\wedge \rho}{\vee \rho-\wedge \rho} .
$$

where $\vee \rho=\max _{v_{i}} \rho\left(v_{i}\right)$ and $\wedge \rho=\min _{v_{i}} \rho\left(v_{i}\right)$ respectively denote the maximum and minimum values of $\rho\left(v_{i}\right)$. 
3) proposed adaptation: Using the operators presented in Section II, and the notions of patch and potential on graphs, we propose the following adaptation of (20):

$$
\begin{array}{r}
\frac{\delta f\left(v_{i}, t\right)}{\delta t}=\delta_{\varepsilon}\left(f\left(v_{i}, t\right)\right)\left[\mu g\left(v_{i}\right)\left(\kappa_{w} f\right)\left(v_{i}, t\right)\right. \\
+\mu \nabla g\left(v_{i}\right) \cdot\left(\nabla_{w} f\right)\left(v_{i}, t\right)+v g\left(v_{i}\right) \\
\left.-\frac{\lambda_{1}}{d} \sum_{v_{i}} d^{2}\left(\mathcal{P}_{\beta}\left(f_{I}, v_{i}\right), \mathcal{P}_{\beta}^{c_{1}}\left(f_{I}\right)\right)+\frac{\lambda_{2}}{d} \sum_{v_{i}} d^{2}\left(\mathcal{P}_{\beta}\left(f_{I}, v_{i}\right), \mathcal{P}_{\beta}^{c_{2}}\left(f_{I}\right)\right)\right]
\end{array}
$$

with $f_{I}$ the initial graph signal and $\mathcal{P}_{\beta}^{c_{1}}\left(f_{I}\right)=$ average $\left\{\mathcal{P}_{\beta}\left(f_{I}, v_{i}\right): f\left(v_{i}\right) \geq 0\right\} \quad$ and $\mathcal{P}_{\beta}^{c_{2}}\left(f_{I}\right)=$ average $\left\{\mathcal{P}_{\beta}\left(f_{I}, v_{i}\right): f\left(v_{i}\right)<0\right\}$. This means that we consider average patch-based models to represents the regions and not only their average values (recovered when $\beta=0$ ). As in Eq. (22), we can express the front propagation as a combinaison of morphological gradients. We introduce a new speed function defined as

$$
\begin{array}{r}
\mathcal{F}\left(v_{i}, t\right)=v g\left(v_{i}\right)+\mu g\left(v_{i}\right)\left(\kappa_{w} f\right)\left(v_{i}, t\right) \\
-\frac{\lambda_{1}}{d} \sum_{v_{i}} d^{2}\left(\mathcal{P}_{\beta}\left(f_{I}, v_{i}\right), \mathcal{P}_{\beta}^{c_{1}}\left(f_{I}\right)\right)+\frac{\lambda_{2}}{d} \sum_{v_{i}} d^{2}\left(\mathcal{P}_{\beta}\left(f_{I}, v_{i}\right), \mathcal{P}_{\beta}^{c_{2}}\left(f_{I}\right)\right)
\end{array}
$$

This is an extension of the speed function proposed in [14] to include balloon and smoothing forces, weighted by the potential $g$, and with patch-based models to represent regions. Then the front propagation can be expressed as

$$
\begin{aligned}
\frac{\delta f\left(v_{i}, t\right)}{\delta t}= & \delta_{\varepsilon}\left(f\left(v_{i}\right)\right)\left[\mu \nabla g\left(v_{i}\right) \cdot\left(\nabla_{w} f\right)\left(v_{i}, t\right)\right. \\
& +\max \left(\mathcal{F}\left(v_{i}, t\right), 0\right)\left\|\left(\nabla_{w}^{+} f\right)\left(v_{i}\right)\right\|_{p}^{p} \\
& \left.+\min \left(\mathcal{F}\left(v_{i}, t\right), 0\right)\left\|\left(\nabla_{w}^{-} f\right)\left(v_{i}\right)\right\|_{p}^{p}\right]
\end{aligned}
$$

The time variable is discretized using the explicit Euler method as $\frac{\delta f\left(v_{i}\right.}{\delta t}=\frac{f^{t+1}\left(v_{i}\right)-f^{t}\left(v_{i}\right)}{\Delta t}$. The general iterative scheme to compute $f$ at time $t+1$ at a given vertex $v_{i}$ is given by

$$
\begin{aligned}
f^{t+1}\left(v_{i}\right)=f^{t}\left(v_{i}\right) & +\Delta t \delta_{\varepsilon}(f)\left[\mu \sum_{v_{j} \sim v_{i}}\left(d_{w} g\right)\left(v_{i}, v_{j}\right)\left(d_{w} f\right)\left(v_{i}, v_{j}\right)\right. \\
& +\max \left(\mathcal{F}\left(v_{i}, t\right), 0\right)\left\|\left(\nabla_{w}^{+} f\right)\left(v_{i}\right)\right\|_{p}^{p} \\
& \left.+\min \left(\mathcal{F}\left(v_{i}, t\right), 0\right)\left\|\left(\nabla_{w}^{-} f\right)\left(v_{i}\right)\right\|_{p}^{p}\right]
\end{aligned}
$$

Given the front $\Omega_{t}$ at time step $t$, its inner boundary set is $\partial^{-} \Omega_{t}=\left\{v_{i} \in \overline{\Omega_{t}}: \exists v_{j} \in \Omega_{t}\right.$ with $\left.\left(v_{i}, v_{j}\right) \in \mathcal{E}\right\}$ and its outer boundary set is $\partial^{+} \Omega_{t}=\left\{v_{i} \in \Omega_{t}: \exists v_{j} \in \overline{\Omega_{t}}\right.$ with $\left.\left(v_{i}, v_{j}\right) \in \mathcal{E}\right\}$. Since the $\left(d_{w} f\right)\left(v_{i}, v_{j}\right)$ are equal to zero on the contant areas of $f$, the set of vertices to be updated at each iteration can be restricted to $\partial^{-} \Omega_{t} \cup \partial^{+} \Omega_{t}$. This means that at each iteration only the vertices around the narrow band of the front have to be considered. In addition, we reinitialize the level set function $f$ every ten iterations by $f=\min (\max (f,-1), 1)$. Both these tricks enable to speed up much the computing (convergence can be obtained in around 100 iterations). The final solution is given by the zero-level of the level-set function $f^{t}\left(v_{i}\right)$.
The proposed formulation is valid for any $p$. However, for $p=1$, specific formulations can be derived thanks to the coarea formula on graphs [15] with a convex formulation and faster algorithms can be considered [23].

\section{EXPERIMENTS}

To illustrate the proposed approach (named GSAC), we consider different types of graph signals : color images (color signals on grid graphs), colored 3D meshes (color signals on triangular meshes), and image databases. We fix some parameters values: $\varepsilon=1, \mu=1, \lambda_{1}=\lambda_{1}=1, v=-1, p=2$. We also set $\Delta t=\frac{1}{\max _{v_{i}} \operatorname{deg}\left(v_{i}\right)}$. To terminate the iterations, we stop when the $L_{2}$ difference between $f^{t+1}$ and $f^{t}$ is below $10^{-2}$ and do not go beyond 100 iterations. The weights of the graphs are obtained by

$$
w\left(v_{i}, v_{j}\right)=\exp \left(\frac{-d\left(\mathcal{P}_{\beta}\left(f_{I}, v_{i}\right), \mathcal{P}_{\beta}\left(f_{I}, v_{j}\right)\right)^{2}}{\sigma^{2}}\right)
$$

where $d$ is $L_{2}$ distance and $f_{I}$ the original graph signal.

\section{A. Grid graph signals}

First we consider the classical case of color images that are represented as 8-adjacency grid graphs, giving graph signals $f_{I}: \mathcal{V} \rightarrow \mathbb{R}^{3}$. We compare our model with the classical CV method [11] (using the implementation of [21]), and the $\mathrm{CV}$ adaption on graphs of [22]. We consider a checkerboard initialization [21]. The classical $\mathrm{CV}$ approach fails to extract the bird, whereas ours (with $g\left(v_{i}\right)=1, \beta=0$ ) does succeed and provide similar results to [22] (as them, we also consider Lab values instead of RGB values). If we consider the use of non constant potential function $g$ with $\alpha=2(5 \times 5$ patches $)$ and $k=7$ (a neighborhood of size $15 \times 15$ ), small spurious regions can be removed. Finally if we consider $\beta=1$, which means the use of patches to represent the regions and weight the graph, a much more smooth contour is obtained, showing the benefit of our whole formulation. Figure 2 provides a comparison with the nonlocal active contours (NLAC) of [24]. We can obtain competitive results with this approach. However this is at the cost of using larger patches with $\beta=5(11 \times 11$ patches $)$ whereas in [24] patches of size $7 \times 7$ are considered. Figure 3 presents the results obtained on three different images, with the same parameters and $\beta=2$, starting from a checkboard initialization. As it can be seen, our approach can successfully extract the objects of interest in the images. The last image of Figure 3 is from the Bayeux Tapestry ${ }^{1}$.

\section{B. $3 D$ colored meshes}

Second we consider the more challenging case of 3D colored meshes, where the graph is a triangular mesh and colors are associated to vertices giving a graph signal $f_{I}: \mathcal{V} \rightarrow \mathbb{R}^{3}$. The graph is irregular and vertices can have a different number of neighbors, leading to patches of different sizes that are therefore not directly comparable with a $L_{2}$ norm. To cope

\footnotetext{
${ }^{1}$ Panorama of the Bayeux Tapestry, GREYC Image (UMR UNICAEN/ENSICAEN/CNRS), CERTIC (UNICAEN) from the 2017 photographic campaign of La Fabrique de patrimoines en Normandie, 2017-2018.
} 


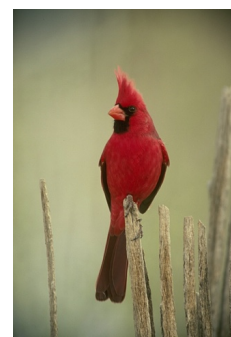

(a)

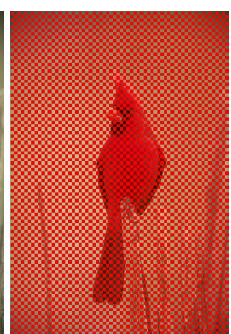

(b)

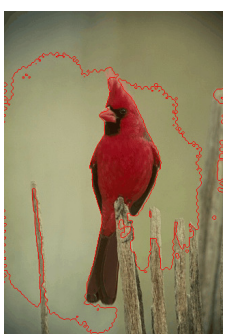

(c)

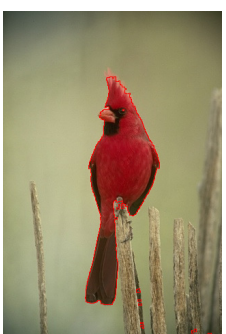

(d)

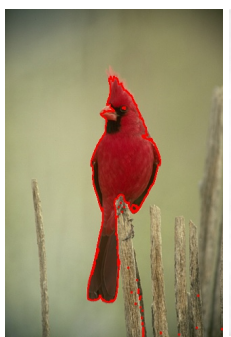

(e)

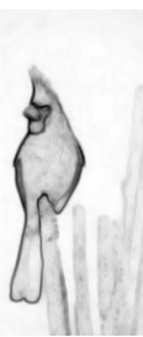

(f)

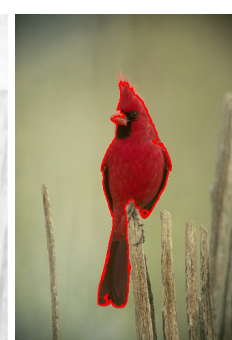

(g)

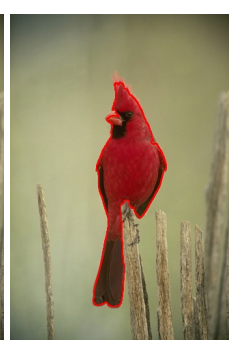

(h)

Fig. 1. Comparison between the state-of-the-art and our approach with different parameters. From left to right: (a) Original image, (b) Checkerboard initialization, (c) [21], (d) [22], (e) GSAC; $g\left(v_{i}\right)=1, \beta=0$, (f) $g\left(v_{i}\right)$, (g) GSAC; $g\left(v_{i}\right), \beta=0$, (h) GSAC; $g\left(v_{i}\right), \beta=1$.

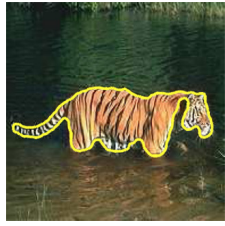

NLAC

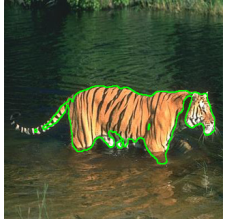

GSAC

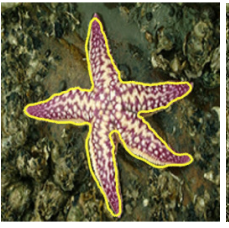

NLAC

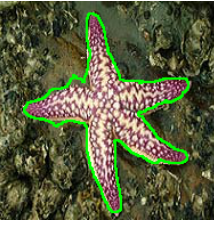

GSAC
Fig. 2. Comparison between NLAC and our approach.
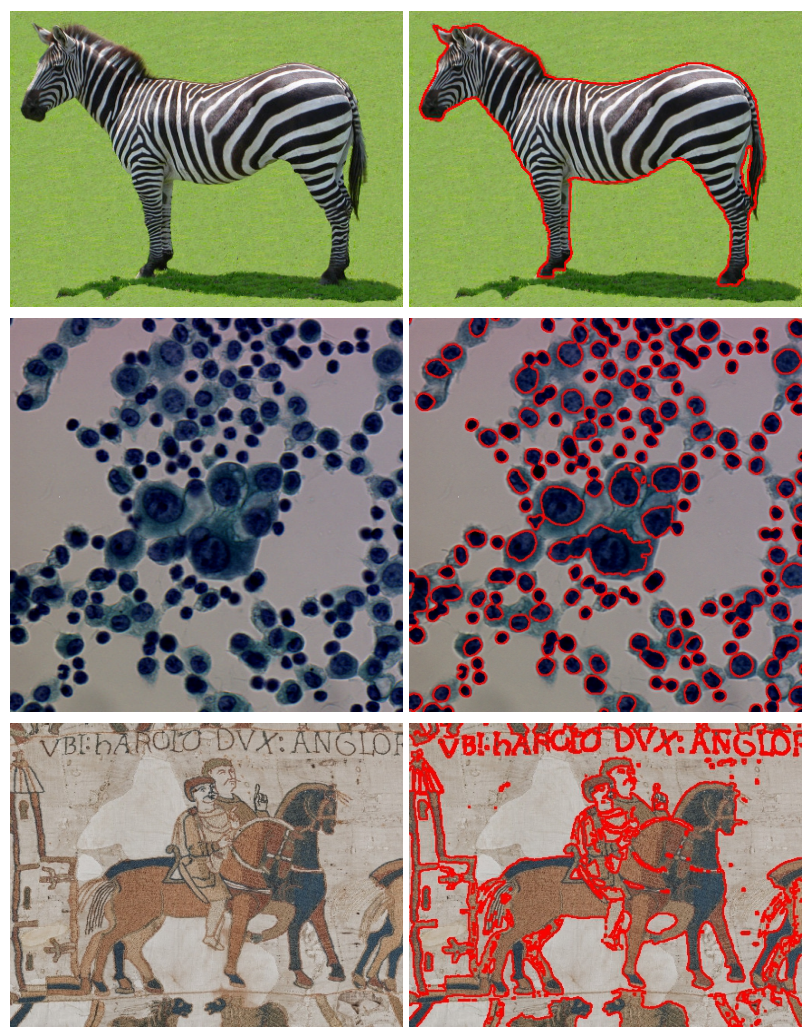

Fig. 3. Results of our GSAC on various types of images. with this, the distance $d$ to compare the patches is the Earth Mover Distance [25] between the color histograms of the patches (with colors in the Lab color space as for images). This distance is used in (30) instead of the $l_{2}$ norm. We compute the potential $g$ with $\alpha=2$ and $k=1$. Figure 4 presents a small size Mesh from [26] and the computed potential (inverted for visualization). Then two results obtained with our GSAC approach from a checkerboard initialization are presented: for $\beta=0$ and $\beta=2$. One can see that, as for images, the use of patches to weight the graph and represent regions provides better results (the red parts of the mesh have been isolated in one single phase without the remaining small regions obtained without patches). The last three results present how a given region (Mario's pants) can be extracted from a manual initialization and recolored. This shows the interest of our approach for mesh editing applications. There are very few competing methods. As far as we know, only the method of Lozes et al. [23] proposes nonlocal active contours on graphs. In Figure 5, we provide a comparison with their results. As it can be seen, we obtain similar results with the same initialization. In our case we extract only one region since our approach is not nonlocal. Figure 6 presents a last segmentation result on a very large mesh (3.5 Million of vertices) obtained from a checkerboard initialization. We were able to accurately extract the different visual components of the mesh.

\section{Image dataset graph}

To end up these experiments, we consider a last type of graph. Given a dataset of images, a $\tau$-nearest neighbor graph is built. To compare images (assumed of the same size), we use a $L_{2}$ norm. The weights are defined as

$$
w\left(v_{i}, v_{j}\right)=1-\frac{\left\|f\left(v_{i}\right)-f\left(v_{j}\right)\right\|_{2}}{\max _{v_{k} \sim v_{l} \in \varepsilon}\left\|f\left(v_{k}\right)-f\left(v_{l}\right)\right\|_{2}} .
$$

Once the graph has been constructed, we use an alternate initialization into two classes: vertices of even indexes are assigned to the first class, and vertices of odd indexes to the other class. The first dataset we consider is composed of the first two classes of the Outex 13 color textures dataset [27] (since it contains several classes and we can obtain only two separated classes with our approach). The graph signal is $f_{I}: \mathcal{V} \rightarrow \mathbb{R}^{3 \times 128^{2}}$. With $\tau=5, \lambda_{1}=\lambda_{2}=0.5, k=1$, and $\alpha=0$, 


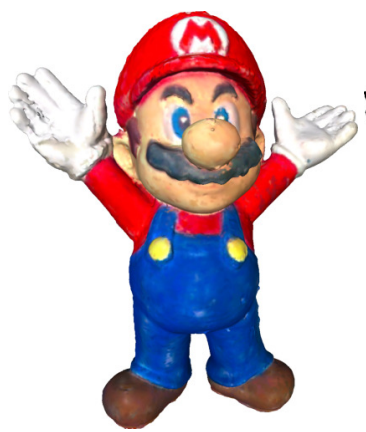

(a)

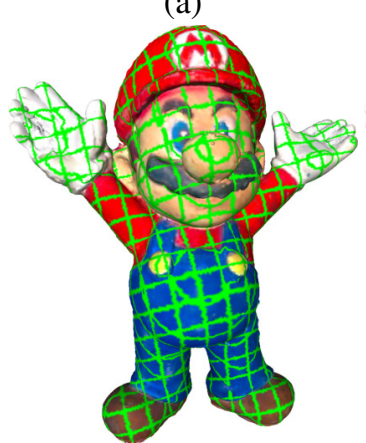

(c)

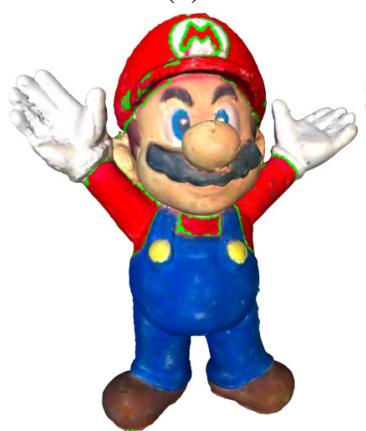

(e)

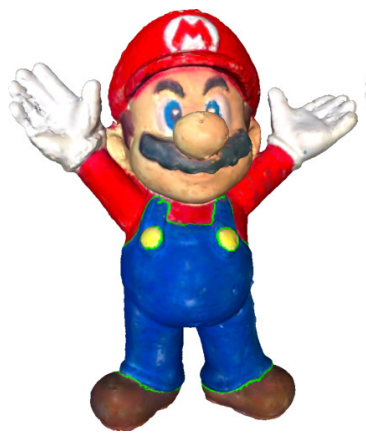

(g)

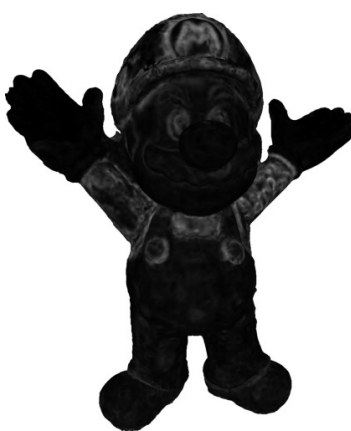

(b)

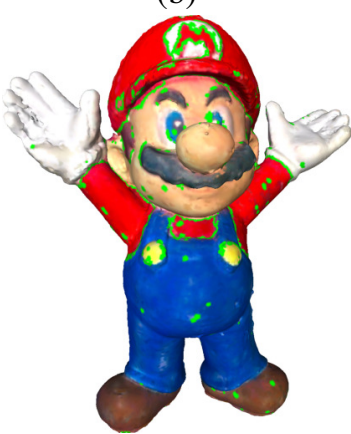

(d)

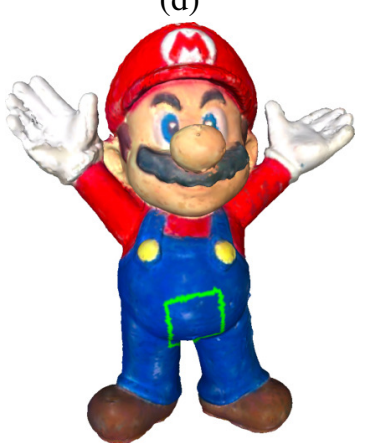

(f)

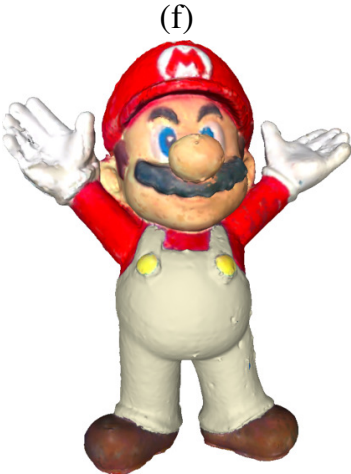

(h)
Fig. 4. From top to bottom, left to right : (a) Original mesh, (b) $g\left(v_{i}\right)$ (inverted) (c) Checkerboard initialization, (d) GSAC; $g\left(v_{i}\right), \beta=0$, (e) GSAC; $g\left(v_{i}\right)$ $\beta=2$, (f) manual initialization (g) extracted region with GSAC; $g\left(v_{i}\right), \beta=2$, (h) re-colorisation of the extracted region.

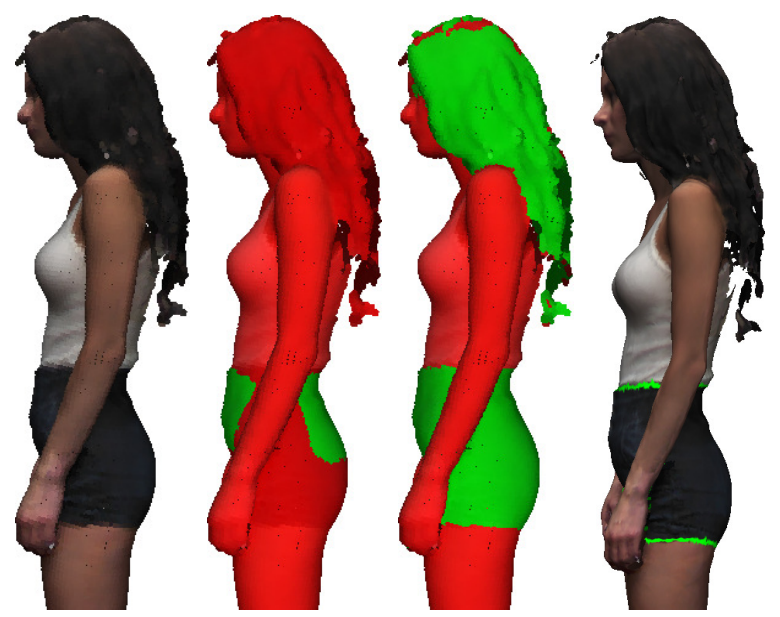

Fig. 5. Comparison between [23] and our approach. From left to right: original mesh, seeds, final result of [23], our result.

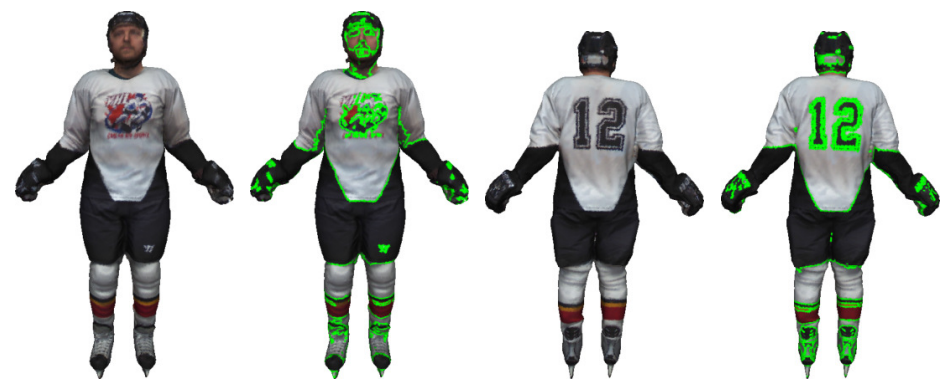

Fig. 6. Segmentation of a large mesh from a checkerboard initialization.

we obtain a classification rate of $100 \%$. Figure 7 presents the graph at initialization and after classification. This (toy) example shows that our approach can be efficient to perform classification. We have also considered subsets of the MNIST dataset with only the 0 digit and another digit. The graph signal is $f_{I}: \mathcal{V} \rightarrow \mathbb{R}^{28^{2}}$. We consider the same random initialization, with parameters $\tau=2, \lambda_{1}=\lambda_{2}=0.5, k=1$, and $\alpha=0$. For the 0 digit versus the 3 digit, we reach a classification rate of $97 \%$. Figure 8 presents a part of the graph at initialization and after classification (the graph is too large to be displayed as a whole). Table I shows the results for all the pairwise combination between digit 0 and other digits. As it can be seen, classification rates are always higher than $90 \%$. These results could be improved by using the two-sided tangent distance [28] between the images instead of a simple $L_{2}$ distance.

\begin{tabular}{|c|c|c|c|c|c|c|c|c|}
\hline 1 & 2 & 3 & 4 & 5 & 6 & 7 & 8 & 9 \\
\hline 98.8 & 95.8 & 97 & 97.05 & 90,7 & 95.55 & 96.75 & 95.95 & 96.25 \\
\hline
\end{tabular}

CLASSIFICATION SCORES FOR THE 0 DIGIT VERSUS EACH OTHER DIGIT OF THE MNIST DATABASE.

\section{CONCLUSION}

In this paper, we have proposed an adaptation of active contours on graphs that combines the Geodesic Active Contour 

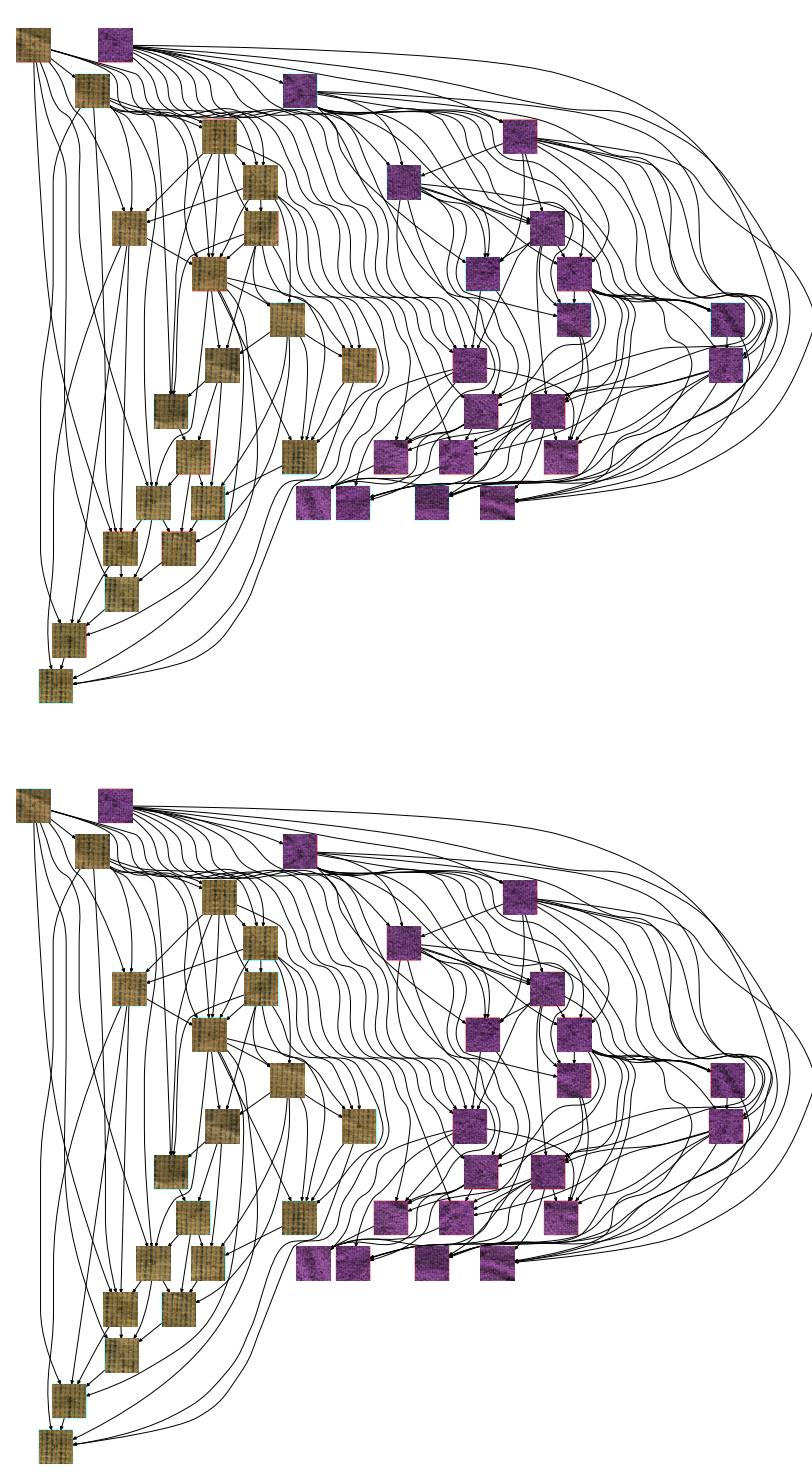

Fig. 7. Classification of a subset of Outex13 dataset. Top: initialization, bottom: final classification. The classes are shown with red and cyan rectangles around each image.

and Active Contours Without Edges approaches. A levelset formulation is adapted on graphs with a framework of graph operators that can describe the evolution of a front on a graph. The proposed approach incorporates specific graph features extracted in the form of a potential function and local graph patches to enhance the segmentation. Various results and comparison with state-of-the-art approaches have shown the benefit of the approach on different types of graphs.

\section{REFERENCES}

[1] A. Ortega, P. Frossard, J. Kovacevic, J. M. F. Moura, and P. Vandergheynst, "Graph signal processing: Overview, challenges, and applications," Proceedings of the IEEE, vol. 106, no. 5, pp. 808-828, 2018.

[2] D. I. Shuman, S. K. Narang, P. Frossard, A. Ortega, and P. Vandergheynst, "The emerging field of signal processing on graphs: Ex-
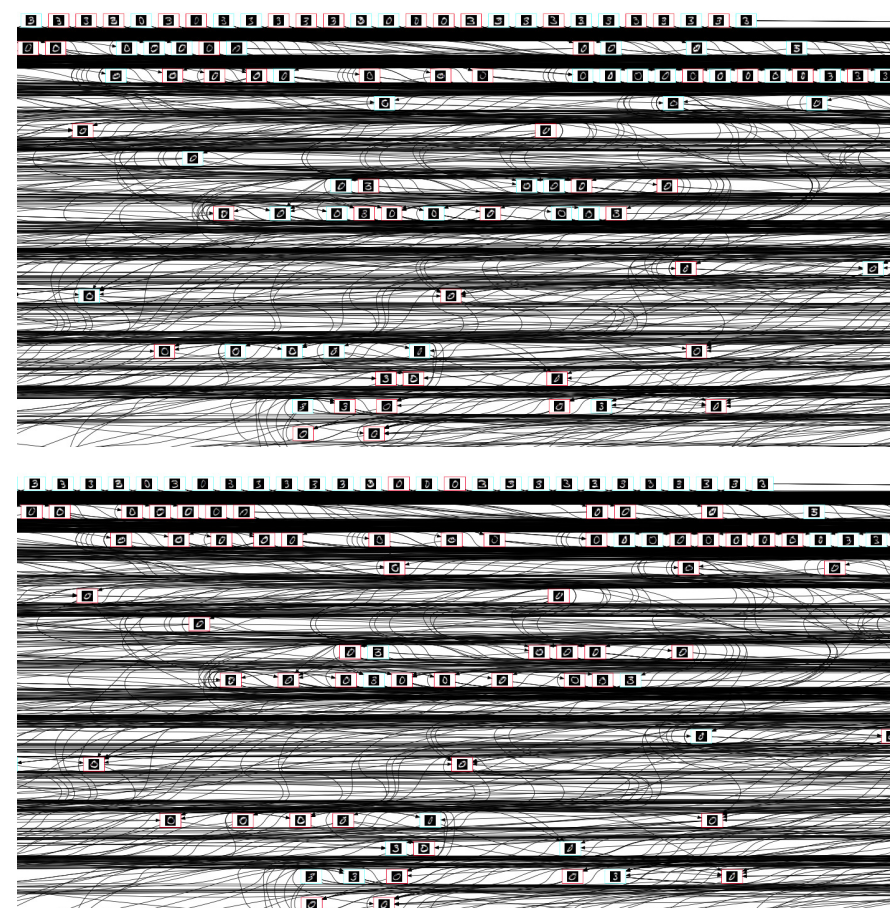

Fig. 8. Classification of a subset (digits 0 and 3) of the MNIST dataset. The colors around each image show the class it is affected to. The top row shows the initialization and bottom second row the final classification.

tending high-dimensional data analysis to networks and other irregular domains," IEEE Signal Process. Mag., vol. 30, no. 3, pp. 83-98, 2013.

[3] D. K. Hammond, P. Vandergheynst, and R. Gribonval, "Wavelets on graphs via spectral graph theory," Applied and Computational Harmonic Analysis, vol. 30, no. 2, pp. 129 - 150, 2011.

[4] _ The Spectral Graph Wavelet Transform: Fundamental Theory and Fast Computation. Springer International Publishing, 2019, pp. 141175.

[5] L. Grady and C. V. Alvino, "The piecewise smooth mumford-shah functional on an arbitrary graph," IEEE Trans. Image Processing, vol. 18, no. 11 , pp. 2547-2561, 2009.

[6] A. Elmoataz, O. Lezoray, and S. Bougleux, "Nonlocal discrete regularization on weighted graphs: A framework for image and manifold processing," IEEE Trans. Image Processing, vol. 17, no. 7, pp. 10471060, 2008.

[7] O. Lézoray and L. Grady, Image Processing and Analysis with Graphs: Theory and Practice, ser. Digital Imaging and Computer Vision, O. Lézoray and L. Grady, Eds. CRC Press / Taylor and Francis, 2012.

[8] V. Ta, A. Elmoataz, and O. Lezoray, "Nonlocal pdes-based morphology on weighted graphs for image and data processing," IEEE Trans. Image Processing, vol. 20, no. 6, pp. 1504-1516, 2011.

[9] _ _Partial difference equations over graphs: Morphological processing of arbitrary discrete data," in Computer Vision - ECCV 2008, 10th European Conference on Computer Vision, Marseille, France, October 12-18, 2008, Proceedings, Part III, ser. Lecture Notes in Computer Science, D. A. Forsyth, P. H. S. Torr, and A. Zisserman, Eds., vol. 5304. Springer, 2008, pp. 668-680.

[10] V. Caselles, R. Kimmel, and G. Sapiro, "Geodesic active contours," International Journal of Computer Vision, vol. 22, no. 1, pp. 61-79, 1997.

[11] T. F. Chan and L. A. Vese, "Active contours without edges," IEEE Trans. Image Processing, vol. 10, no. 2, pp. 266-277, 2001.

[12] G. Sapiro, "Color snakes," Computer Vision and Image Understanding, vol. 68, no. 2, pp. 247-253, 1997.

[13] L. D. Cohen, "On active contour models and balloons," CVGIP: Image Understanding, vol. 53, no. 2, pp. 211-218, 1991.

[14] X. Desquesnes, A. Elmoataz, and O. Lezoray, "Pdes level sets on weighted graphs," in 18th IEEE International Conference on Image 
Processing, ICIP 2011, Brussels, Belgium, September 11-14, 2011, 2011, pp. 3377-3380.

[15] O. Lézoray, A. Elmoataz, and V. Ta, "Nonlocal pdes on graphs for active contours models with applications to image segmentation and data clustering," in 2012 IEEE International Conference on Acoustics, Speech and Signal Processing, ICASSP 2012, Kyoto, Japan, March 2530, 2012, 2012, pp. 873-876.

[16] K. Drakopoulos and P. Maragos, "Active contours on graphs: Multiscale morphology and graphcuts," J. Sel. Topics Signal Processing, vol. 6, no. 7, pp. 780-794, 2012.

[17] R. Kimmel, "Fast edge integration," in Geometric Level Set Methods in Imaging, Vision and Graphics, S. Osher and N. Paragios, Eds. Springer Verlag, 2003, pp. 59-77.

[18] L. Pi, Y. Peng, C. Shen, and F. Li, "A modified chan-vese model and its theoretical proof," Journal of Mathematical Analysis and Applications, vol. 351, no. 2, pp. $627-634,2009$.

[19] V. Caselles, F. Catté, T. Coll, and F. Dibos, "A geometric model for active contours in image processing," Numerische Mathematik, vol. 66, no. 1, pp. 1-31, Dec 1993.

[20] L. Chen, Y. Zhou, Y. Wang, and J. Yang, "GACV: geodesic-aided C-V method," Pattern Recognit., vol. 39, no. 7, pp. 1391-1395, 2006.

[21] P. Getreuer, "Chan-vese segmentation," IPOL Journal, vol. 2, pp. 214 $224,2012$.

[22] C. Sakaridis, K. Drakopoulos, and P. Maragos, "Theoretical analysis of active contours on graphs," SIAM J. Imaging Sciences, vol. 10, no. 3, pp. 1475-1510, 2017.

[23] F. Lozes, M. Hidane, A. Elmoataz, and O. Lézoray, "Nonlocal segmentation of point clouds with graphs," in IEEE Global Conference on Signal and Information Processing (GlobalSIP - IEEE), 2013.

[24] M. Jung, G. Peyré, and L. D. Cohen, "Nonlocal active contours," SIAM J. Imaging Sciences, vol. 5, no. 3, pp. 1022-1054, 2012.

[25] Y. Rubner, C. Tomasi, and L. J. Guibas, "The earth mover's distance as a metric for image retrieval," International Journal of Computer Vision, vol. 40, no. 2, pp. 99-121, 2000.

[26] A. Nouri, C. Charrier, and O. Lézoray, "Greyc 3d colored mesh database," Technical Report hal-01441721, Tech. Rep., 2017.

[27] T. Ojala, T. Maenpaa, M. Pietikainen, J. Viertola, J. Kyllonen, and S. Huovinen, "Outex - new framework for empirical evaluation of texture analysis algorithms," in Pattern Recognition, 2002. Proceedings. 16th International Conference on, vol. 1, 2002, pp. 701-706 vol.1.

[28] D. Keysers, J. Dahmen, T. Theiner, and H. Ney, "Experiments with an extended tangent distance," in 15th International Conference on Pattern Recognition, ICPR'00, Barcelona, Spain, September 3-8, 2000. IEEE Computer Society, 2000, pp. 2038-2042.

\section{ACKNOWLEDGMENTS}

This work received funding from Saint-Lo Agglo under the TP2SL project. We thank the DSI of Unicaen for providing the image of the Bayeux Tapestry. 